\title{
A Relaxation-Oscillator-Based Interface for High-Accuracy Ratiometric Signal Processing of Differential-Capacitance Transducers
}

\author{
Kouji Mochizuki, Kenzo Watanabe, Fellow, IEEE, Takashi Masuda, and Motoyasu Katsura
}

\begin{abstract}
For high-accuracy signal processing of differentialcapacitance transducers, an interface circuitry is developed based on a relaxation oscillator. Two capacitors of the transducer are multiplexed by diode switches to form the op-amp-based integrator. The duty ratio of the oscillator output then measures the ratio of two capacitances. A circuit analysis shows that the interface can detect the capacitance change as small as $0.01 \%$ of the total capacitance. Experimental results are also given to confirm the analysis.
\end{abstract}

Index Terms-Analog circuit, capacitive transducer, intelligent transducer, pressure measurement, relaxation oscillator, signal processing.

\section{INTRODUCTION}

D IFFERENTIAL capacitance transducers consisting of ganged two capacitors are widely used for detecting pressure difference, linear displacement, and rotational angle [1], [2]. Their principles of detection are based on the complementary changes of two capacitors with the measurand. The capacitance change may be linear or nonlinear. In either case, the accurate and linear representation of the measurand can be obtained by dividing the capacitance difference between two capacitors by their sum. Several techniques have so far been proposed for such a ratiometric signal processing, including switched-capacitor analog-to-digital (A/D) [3], [4], capacitance-to-frequency [5], [6], capacitance-to-phase [7], and capacitance-to-voltage conversion [8].

This paper describes yet another simple interface for the ratiometric signal processing. The interface is basically a relaxation oscillator. Such a configuration has been applied widely to capacitive sensors to detect their capacitance change in the frequency form [5], [6]. Different from these applications, the interface presented here detects the ratio of two capacitances in the form of the duty ratio. This signal processing in the time domain allows high-speed measurements. In the followings, the interface configuration, the ratiometric operation, the accuracy estimate, and experimental results will be described.

Manuscript received June 1, 1997; revised January 1, 1998.

K. Mochizuki and M. Katsura are with the Numazu College of Technology, Numazu, 410 Japan.

K. Watanabe is with the Research Institute of Electronics, Shizuoka University, Hamamatsu, 432 Japan.

T. Masuda is with the Engineering Department, Yamatake-Honeywell Co. Ltd., Fujisawa, 251 Japan.

Publisher Item Identifier S 0018-9456(98)05473-4.

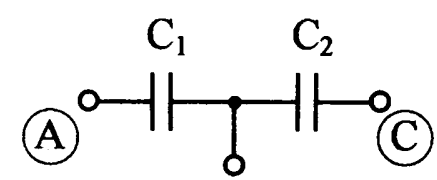

(B)

Fig. 1. An equivalent circuit of a differential capacitance transducer.

\section{TRANSDUCER MODEL}

A differential capacitance transducer can be represented by two capacitors with a common electrode, as shown in Fig. 1 [8].

In a linear displacement encoder, the areas of the electrodes change linearly with the displacement $x$. The capacitances $C_{1}$ and $C_{2}$ can then be expressed as follows:

$$
C_{1,2}=\frac{C_{0}}{2}(1 \pm x)
$$

where $C_{0}$ is a total capacitance of the transducer. In a differential pressure transducer, on the other hand, the spacings between the electrodes change linearly with the pressure difference $x$. Therefore, $C_{1}$ and $C_{2}$ are expressed as

$$
C_{1,2}=\frac{C_{0}}{2} \frac{1}{1 \mp x} \text {. }
$$

Whether the capacitance change is linear or hyperbolic, the measurand $x$ can be detected independently of the total capacitance $C_{0}$ by the following ratiometric operation:

$$
x=\frac{C_{1}-C_{2}}{C_{1}+C_{2}}=\frac{2 C_{1}}{C_{1}+C_{2}}-1 .
$$

\section{INTERFACE CIRCUITRY}

The circuit diagram of the interface circuit to perform the ratiometric operation is shown in Fig. 2. The interface consists of an integrator followed by a comparator. The output of the comparator is fed back to the integrator to sustain the relaxation oscillation.

Two capacitors $C_{1}$ and $C_{2}$ of a differential capacitance transducer are multiplexed by diodes $D_{1}$ and $D_{2}$ to form the integrator $A_{1}$. The multiplexing is made automatically depending on the outputs $V_{1}$ and $V_{4}$ of the integrator and the comparator, respectively.

For the moment, diodes are assumed to be ideal switches. This assumption is reasonable because the diodes are incorporated into the feedback path of the op-amp $A_{1} . R_{1}=R_{2}=R_{3}$ 


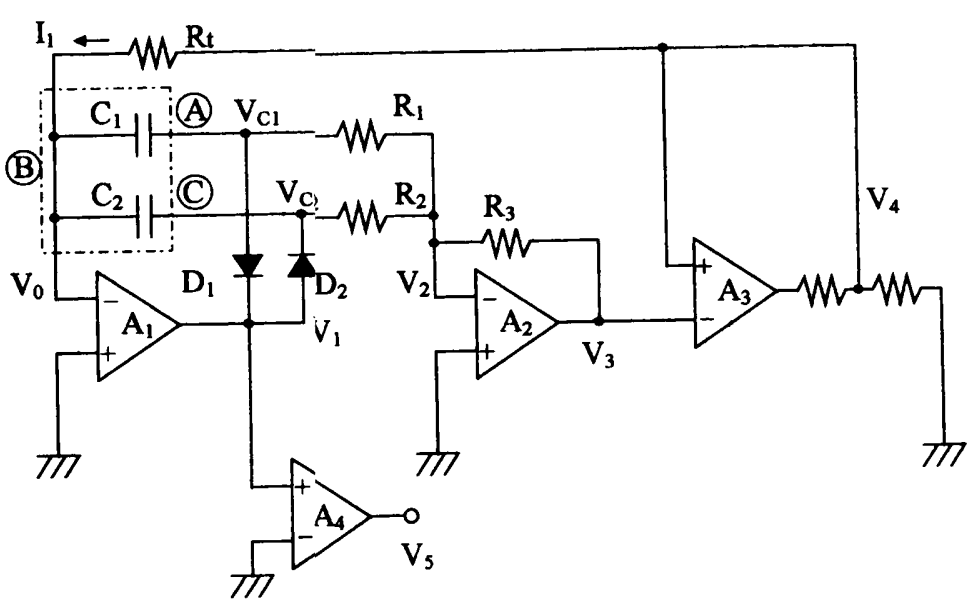

Fig. 2. The circuit dią̧ram of the interface.

is also assumed. Op-amp $A_{2}$ then forms the adder with the weight -1 . The adder operates, in fact, as the inverting voltage follower, because $L_{1}$ and $D_{2}$ never turn on simultaneously.

Depending on the switch conditions, the operation of the oscillator is divided into four states $T_{i}(i=1,2,3,4)$. The integrator and waveforms in each state are shown in Figs. 3 and 4 , respectively.

In the $T_{1}$ state, 'when the output of the comparator is high ( $V_{4}=V_{u}$ ) and the output of op-amp $A_{1}$ is positive, the capacitor $C_{2}$ is charged through $R_{t}$ with the polarity opposite to that shown in Fig. 3(a). The output of $A_{1}$ thus decreases linearly, as shown in Fig. 4. This operation continues until the output of $A_{1}$ is zery and $D_{2}$ is switched off. The initial voltage of $C_{2}$ is $V_{d}$. The duration $T_{1}$ is then given by

$$
T_{1}=C_{2} R_{t} V_{d} / V_{u}
$$

The diode $D_{1}$ is now forward-biased and the operation is switched to the $T_{2}$ state. The integrator on this state charges $C_{1}$ with the polarity shown in Fig. 3(b). This decreases the output of $A_{1}$ until it reaches $-V_{u}$, the threshold voltage of the comparator. $T_{2}$ is thus given by

$$
T_{2}=C_{1} R_{t} \text {. }
$$

$T_{2}$ is independent of the voltage, because the integrator is driven by the threshold voltage of the comparator.

When the outpit of $A_{1}$ reaches the threshold voltage, the output of the comparator changes to $-V_{d}$, and the operation is switched to the $T_{3}$ state. In this state, the integrator charges $C_{1}$ with the polarity shown in Fig. 3(c). This operation continues until the output of $A_{1}$ is zero and $D_{1}$ is switched off. $T_{3}$ is then given by

$$
T_{3}=C_{1} R_{t} V_{u} / V_{d}
$$

When $V_{1}$ reaches zero, $D_{2}$ is now forward-biased, and the operation is switched to the $T_{4}$ state. The integrator in this state charges $C_{2}$ with the polarity shown in Fig. 3(d). This increases the output of $A_{1}$ until it reaches $V_{d}$, the threshold voltage of the comparator. $T_{4}$ is given by

$$
T_{4}=C_{2} R_{t} \text {. }
$$

$T_{4}$ is also independent of the voltage.
The comparator $A_{4}$ performs the triangular-to-rectangular waveform conversion, to produce the output of the interface. The high-level duration $T_{H}$ and the low-level duration of the output pulse are given by

$$
\begin{aligned}
T_{H} & =T_{1}+T_{4}=C_{2} R_{t}\left(V_{d}+V_{u}\right) / V_{u} \\
T_{L} & =T_{2}+T_{3}=C_{1} R_{t}\left(V_{d}+V_{u}\right) / V_{d} .
\end{aligned}
$$

The duty ratio $D$ is thus

$$
D \equiv \frac{T_{H}}{T_{L}+T_{H}}=\frac{C_{2}}{C_{1}+C_{2}}\left(1+\varepsilon_{V}\right)
$$

where

$$
\varepsilon_{V}=\frac{C_{1}\left(1-\frac{V_{u}}{V_{d}}\right)}{C_{1} \frac{V_{u}}{V_{d}}+C_{2}} .
$$

If $V_{u}=V_{d}$, then $\varepsilon_{V}$ is zero and the duty ratio corresponds exactly to the capacitance ratio. The duty ratio can be measured easily by means of gating a high-frequency clock. The measurement is accomplished in one cycle of the output pulse. Therefore, the interface shown in Fig. 2 allows the high-speed signal processing of a differential capacitance transducer. The analog equivalent of the capacitance ratio can also be obtained, if desired, by low-pass filtering the output pulse.

\section{ACCURACY}

Error sources involved in the interface are the voltage unbalance $V_{u} / V_{d}$, mismatch between $R_{1}$ and $R_{2}$, bias current $I_{B}$ of op-amp $A_{1}$, and offset voltages $V_{\text {off }}$ of op-amps and comparators. To eliminate the voltage unbalance effect, the interface is modified as shown in Fig. 5. The modification is based on the fact that $T_{2}$ and $T_{4}$ given by (5) and (7), respectively, do not depend on the voltage. This configuration has another advantage that nonideal performances of diode switches have no effect on the accuracy, either.

Waveforms observed in the improved interface are depicted in Fig. 6. In the figure, $T_{5}$ is defined as the period during which the comparators $A_{3}$ and $A_{4}$ both assume the high level, while $T_{6}$ as the period during which $A_{3}$ and $A_{4}$ both assume the low level. During $T_{5}$, the integrator $A_{1}$ keeps charging $C_{1}$ by the current $\left\{\left(V_{u}-V_{\text {off } 1}\right) / R_{t}-I_{B}\right\}$ and $V_{3}$ slews from $\left(\alpha V_{u}-V_{\text {off } 4}\right)$ up to $\left(V_{u}-V_{\text {off } 3}\right) . T_{5}$ is thus given by

$$
T_{5}=\frac{C_{1} R_{1} R_{t}}{R_{3}} \cdot \frac{(1-\alpha) V_{u}-V_{\text {off } 3}+V_{\text {off } 4}}{V_{u}-V_{\text {off } 1}-R_{t} I_{B}} .
$$

During $T_{6}$, on the other hand, the integrator $A_{1}$ keeps drawing the current $\left\{\left(V_{d}+V_{\text {off } 1}\right) / R_{t}+I_{B}\right\}$ from $C_{2}$ and $V_{3}$ slews from $\left(-\alpha V_{d}-V_{\text {off } 4}\right)$ down to $\left(-V_{d}-V_{\text {off3 } 3}\right) . T_{6}$ is thus given by

$$
T_{6}=\frac{C_{2} R_{2} R_{t}}{R_{3}} \cdot \frac{(1-\alpha) V_{d}-V_{\mathrm{off} 3}+V_{\mathrm{off} 4}}{V_{d}-V_{\mathrm{off} 1}-R_{t} I_{B}} .
$$

From (12) and (13), one can obtain the first order expression of the capacitance ratio

$$
\frac{T_{5}}{T_{5}+T_{6}}=\frac{C_{1}}{C_{1}+C_{2}}(1+\varepsilon)
$$




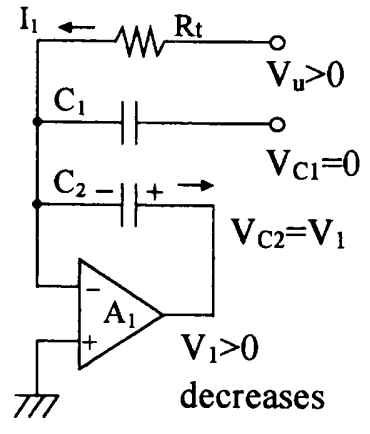

$\mathrm{T}_{1}$

(a)

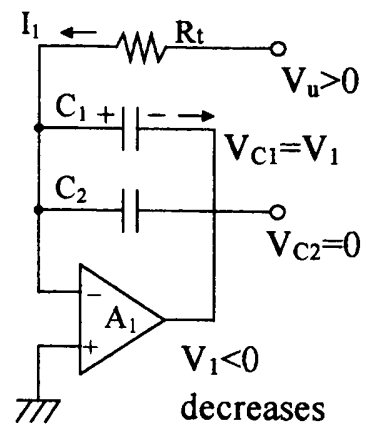

$\mathrm{T}_{2}$

(b)

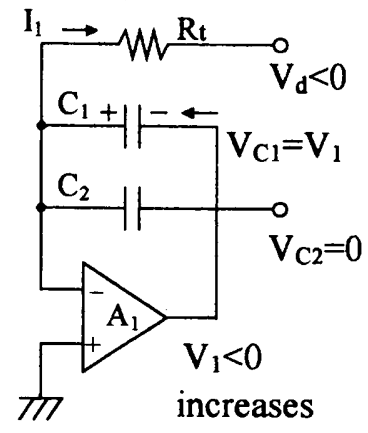

$\mathrm{T}_{3}$

(c)

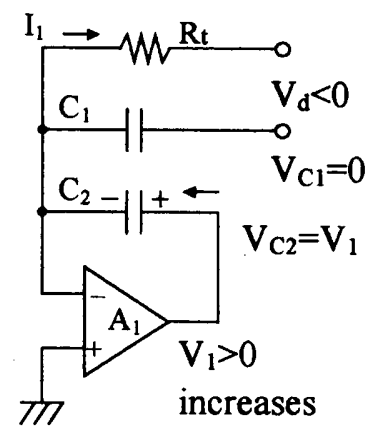

$\mathrm{T}_{4}$

(d)

Fig. 3. The integrator in each state.

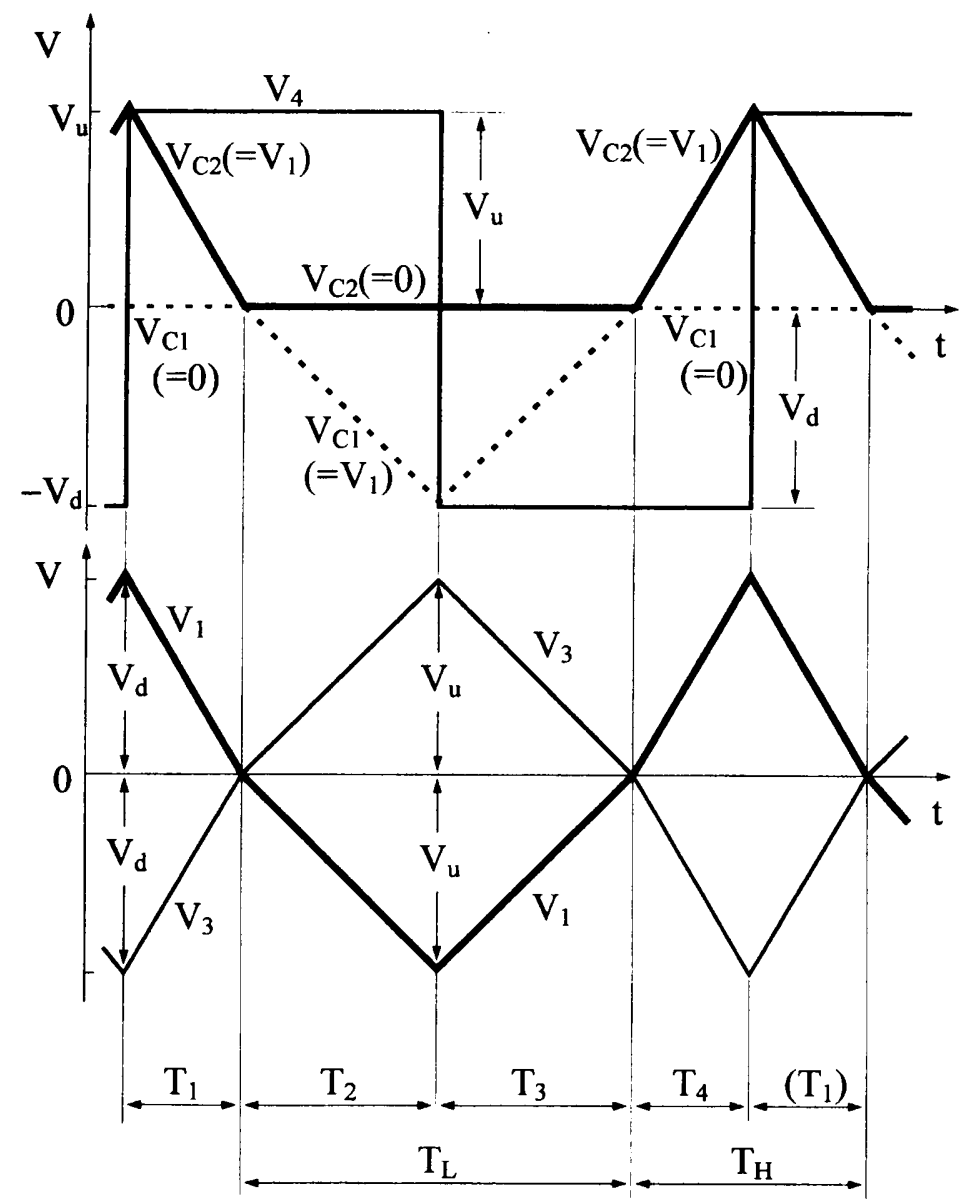

Fig. 4. Waveforms, observed in the interface.

where

$$
\begin{aligned}
\varepsilon= & \frac{C_{2}}{C_{1}+C_{2}} \varepsilon_{R}+\frac{2 C_{2}}{C_{1}+C_{2}} \\
& \cdot\left\{\frac{R_{t} I_{B}}{V_{u}}+\frac{V_{\text {off } 1}}{V_{u}}-\frac{V_{\text {off } 3}-V_{\text {off } 4}}{(1-\alpha) V_{u}}\right\} \\
\varepsilon_{R}= & \frac{R_{1}-R_{2}}{R_{1}} .
\end{aligned}
$$

The first term represents the error due to the resistance mismatch and the second term due to the offset voltages. The error due to each source is evaluated for $x$ ranging from

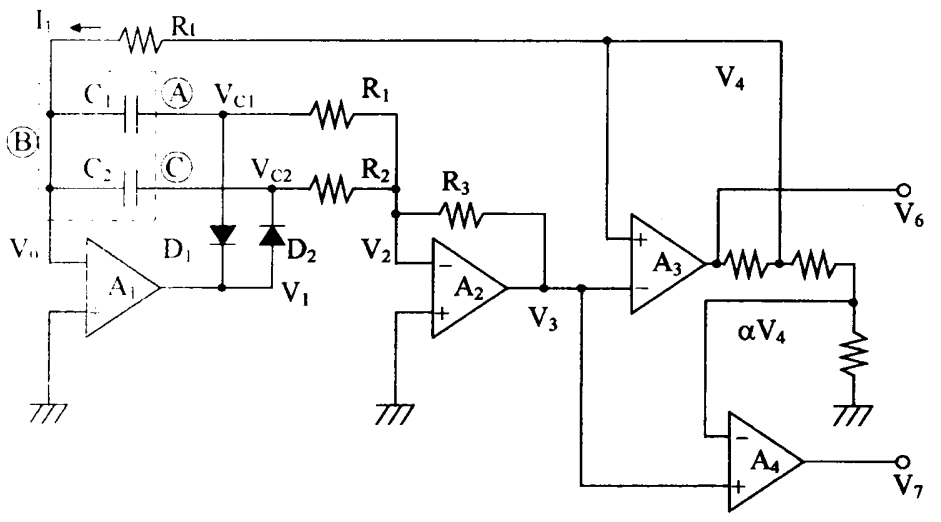

Fig. 5. An improved circuit.

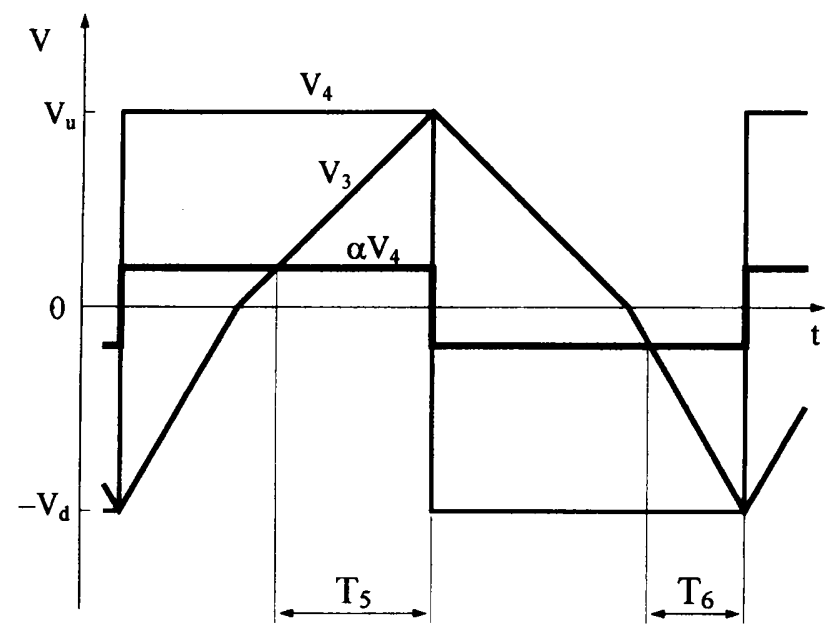

Fig. 6. Waveforms observed in the improved interface.

-0.5 to 0.5 using following typical values; $V_{d}=V_{u}=10 \mathrm{~V}$, $R_{1} \cong R_{2} \cong R_{3}=1 \mathrm{k} \Omega, R_{t}=125 \mathrm{M} \Omega, C_{0}=C_{1}+C_{2}=2$ $\mathrm{pF}, \alpha=0.1$. The results are listed in Table $\mathrm{I}$, indicating that a resolution higher than $0.1 \%$ can be expected from this simple interface.

\section{PROTOTYPE INTERFACE}

A prototype interface based on Fig. 2 was breadboarded using off-the-shelf op-amps (LF411). To evaluate its performance, a ganged parallel plate capacitor was used as a 
TABLE I

ESTIMATED ERRORS

\begin{tabular}{cc}
\hline Error source & $\varepsilon_{\max }$ \\
\hline$\varepsilon_{\mathrm{R}}=1 \times 10^{-3}$ & $0.75 \times 10^{-3}$ \\
$\mathrm{I}_{\mathrm{B}}=15 \mathrm{pA}$ & $0.28 \times 10^{-3}$ \\
$\mathrm{~V}_{\text {off }}=0.3 \mathrm{mV}$ & $0.15 \times 10^{-3}$ \\
\hline
\end{tabular}

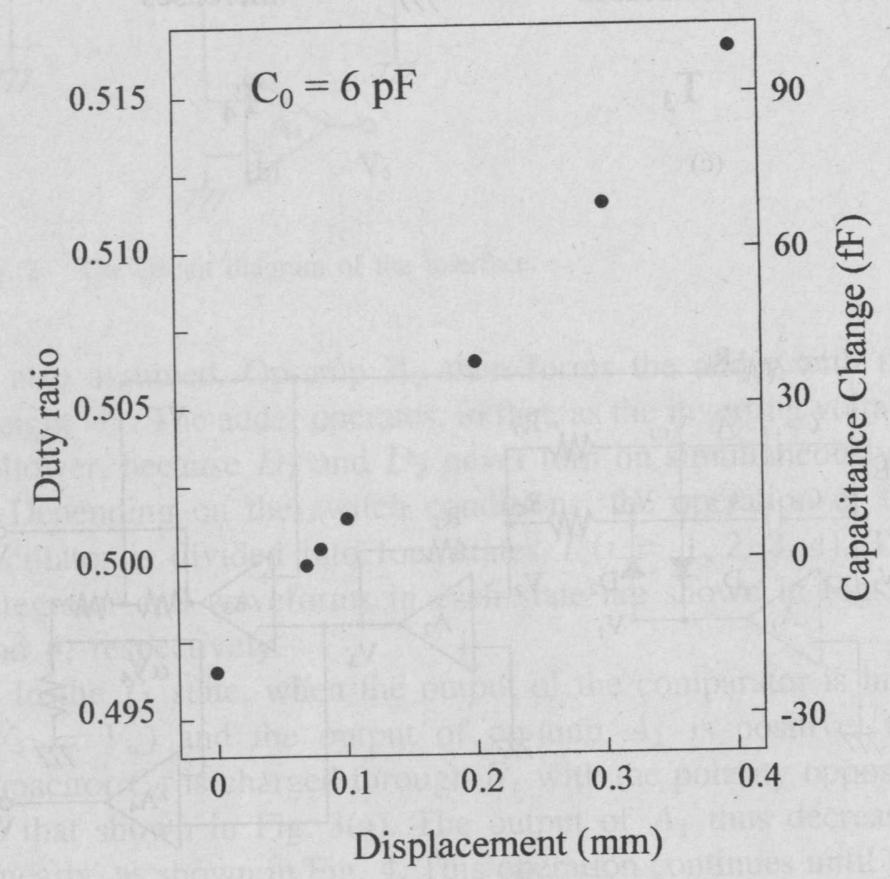

Fig. 7. Experimentally measured capacitance change and duty ratio.

transducer [8]. The $30 \mathrm{MHz}$ clock was used for the duty ratio measurement.

The capacitances $C_{1}$ and $C_{2}$ are given by (2) with $x$ being the displacement of the inner electrode from the center of two outer electrodes. The total capacitance $C_{0}$ is $3 \mathrm{pF}$ or $6 \mathrm{pF}$.

Fig. 7 shows typical measurement results when $C_{0}=6 \mathrm{pF}$. The oscillation frequency when $C_{1}=C_{2}$ is adjusted to be $0.5 \mathrm{kHz}$ by means of $R_{t}$. Dots plotted in the figure are averaged values of 20 measurements at each displacement. The displacement is adjusted by a micrometer screw with the finest scale of $10 \mu \mathrm{m}$.

To evaluate the resolution, measurements are repeated with $C_{0}$ being $3 \mathrm{pF}$. The standard deviation in the capacitance evaluated from the duty ratio measurements is $60 \mathrm{aF}$, which corresponds to $2 \times 10^{-3} \%$ resolution. These results confirm the accuracy estimate in the previous section.

\section{CONCLUSIONS}

An interface circuitry was presented for ratiometric signal processing of a differential capacitance transducer. Circuit analyzes have shown that a resolution higher than $0.1 \%$ is easily achievable with this simple configuration. The prototype interface built using off-the-shelf components has confirmed the circuit analyzes and demonstrated the validity in practical applications.
The ratiometric signal processing is performed in the timedomain by measuring the duty ratio of the output signal. Therefore, the interface proposed here allows high-speed measurements. The sampling speed higher than $5 \mathrm{ksps}$ (samples/s) can be expected from using high-speed comparators. A onechip implementation and applications to practical transducers are future works.

\section{REFERENCES}

[1] T. Saigusa and S. Gotoh, "UNI $\Delta$ series electronic differential pressure transducer," Yokogawa Tech. J., vol. 22, pp. 23-29, Mar. 1978, (in Japanese).

[2] X. Li, G. C. M. Meijer, and G. W. de Jong, "An accurate smart capacitive angular-position sensor with a full circle range," in Instrum Meas. Tech. Conf. Proc., 1995, pp. 80-82.

[3] H. Matsumoto, H. Shimizu, and K. Watanabe, "A switched-capacitor change-balancing analog-to-digital converter and its application to capacitance measurement," IEEE Trans. Instrum. Meas., vol. IM-36, pp 873-878, Dec. 1987.

[4] Y. Cao and G. C. Temes, "High-accuracy circuit for on-chip capacitance ratio testing or sensor readout," IEEE Trans. Circuits Syst., vol. 41, pp. 637-639, Sept. 1994.

[5] T. N. Toth and G. C. M. Meijer, "A low-cost smart capacitive position sensor," IEEE Trans. Instrum. Meas., vol. 41, pp. 1041-1044, Dec. 1992

[6] T. N. Toth, G. C. M. Meijer, and H. M. M. Kerkvliet, "Ultra-linear, low-cost measurement system for multi-electrode pF-range capacitors," in Instrum. Meas. Tech. Conf. Proc., 1995, pp. 512-515.

[7] R. F. Wolffenbuttel and P. P. L. Regtien, "Capacitance-to-phase angle conversion for the detection of extremely small capacities," IEEE Trans. Instrum. Meas., vol. IM-36, pp. 868-872, Dec. 1987.

[8] K. Mochizuki, T. Masuda, and K. Watanabe, "An interface circuit for high-accuracy signal processing of differential-capacitance transducers," in Instrum. Meas. Tech. Conf. Proc., 1996, pp. 1200-1203.

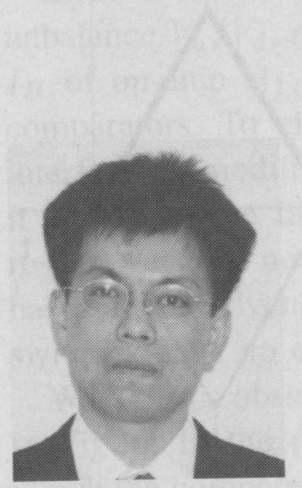

Kouji Mochizuki was born in Shizuoko, Japan, on February 13, 1961. He received the B.E. and M.E. degrees in electronics engineering from Shizuoko University in 1983 and 1985, respectively.

From 1985 to 1989, he was with Fujitsu Laboratories Ltd. where he worked on epitaxial growth of III-V compound semiconductors. Since July 1989 he has been with Namazu College of Technology where he is mainly involved in resistance and capacitance measurements.

Mr. Mochizuki is a member of the Institute of Electronics, Information, and Communication Engineers of Japan.

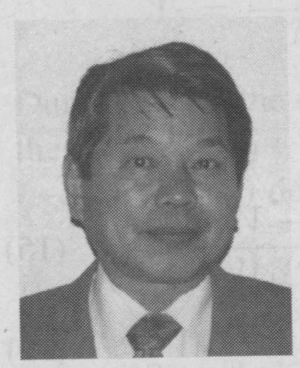

Kenzo Watanabe (M'74-SM'86-F'83) received the B.E. and M.E. degrees in engineering from Shizuoka University, Hamamatsu, Japan, in 1962 and 1966, respectively, and the Dr.Eng. degree from Kyoto University, Kyoto, Japan, in 1976.

$\mathrm{He}$ is a Professor of the Research Institute of Electronics, Shizuoka University. Except for a year as a Visiting Professor at the University of California, Los Angeles, he has been on the faculty at Shizuoka University since 1962, serving progressively as Research Assistant, Associate

Professor, and Professor.

$\mathrm{He}$ serves IEEE activities as an AdCom member of the Instrumentation and Measurement Society, at Associate Editor of the IEEE TRANSACTIONS ON INSTRUMENTATION AND MEASUREMENT, and a member of the IMTC Board of Directors. 


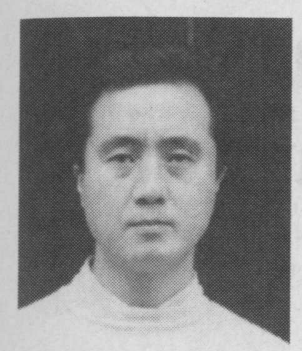

Takashi Masuda was born in Gunma, Japan, on May 5, 1961. He graduated from the Department of Atomic Reactor Engineering, Kinki University, Osaka, Japan, in March 1985. He is now pursuing the Dr.Eng. degree at the Research Institute of Electronics, Shizuoka University, Hamamatsu, Japan.

Since April 1985, he has been with Yamatake Honeywell, Control Products Division, Kanagawa, Japan. Within a division of Yamatake Honeywell he carried out several products in production sensor and sensor signal processing.

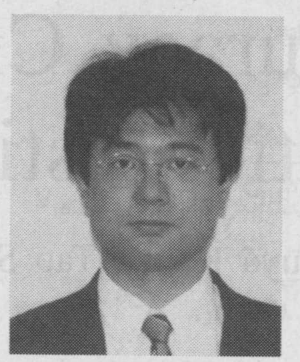

Montoyasu Katsura was born in Shizuoka, Japan, on December 9, 1971. He graduated from the Department of Electrical Engineering, Namazu College of Technology, Namazu, Japan, in March 1992 where in April 1996, he entered an Advanced Engineering course of study.

From 1992 to 1996, he was with OMRON Co. where he worked on programmable controllers.

His research interest is in resistance and capacitance measurements. 